\title{
EDITORIAL
}

\section{Recent Progress in Probability and Statistics in China}

\author{
Dayue CHEN
}

Department of Probability and Statistics, School of Mathematical Sciences, Peking University, Beijing 100871, China

(c) Higher Education Press and Springer-Verlag Berlin Heidelberg 2013

After one year's delay due to the big earthquake in 2011, the Second Institute of Mathematical Statistics Asia Pacific Rim Meeting (IMS-APRM) was held in Tsukuba, Japan, in July of 2012. As a member of the Scientific Committee of the meeting, I was responsible to mobilize participants from China and to recommend invited speakers. My inexperience in fulfilling the task was easily compensated by the enthusiasm of my colleagues. More than 10 statisticians and probabilists from the mainland of China gave invited talks. In particular, Professor Zhi Geng of Peking University delivered a Distinguished Lecture. Recent progress in probability and statistics in China was well presented. When I was asked by the Editor in Chief, Professor K. -C. Chang, to compile a special issue for Frontiers of Mathematics in China, I once again turned to the speakers for help.

The papers collected in this special issue reflect in a fairly honest way the current achievements of Chinese statisticians and probabilists. The ratio of statistics and probability is roughly in accordance with that of the 2nd IMSAPRM meeting. Nowadays the old generation of statisticians faded away and the new generation took over leading roles in every aspect. As you can see, all papers in this special issue were authored by young researchers, most of whom received their Ph. D. degrees in last 15 years or so. Because of an easy access to the world by internet and more academic exchanges, research topics are synchronized with the international trends, although the originality and depth of our work are less satisfactory. Generally speaking, the statistical science has grown quickly in recent years in China, partially because of its wide applications in industrial and commercial practices. The fast growing economy in China sends a strong signal for a huge need of statistics in the near future. A milestone should be noted that the statistical science is now re-classified as a Class I subject by the Ministry of Education, rather than a previous Class II subject named mathematical statistics as a branch of mathematics. The

Received April 1, 2013

Guest editor of this special issue, E-mail: dayue@math.pku.edu.cn 
importance of probability is also better recognized in recent years. The prestigious Fields Medal was awarded to probabilists consecutively through 2006 to 2010, reflecting the general view of mathematicians in the world. I am quite confident that the subject of statistics and probability shall keep a healthy pace to a prosperous phase in China in the years to come.

The year of 2013 was designated as the International Year of Statistics by several statistical societies. It is therefore quite appropriate for Frontiers of Mathematics in China to publish a special issue. I was privileged to serve as a guest editor, and wish to take this opportunity to thank all referees who did a superb job meritoriously and anonymously. Their inputs have significantly improved the manuscripts. 